\title{
Mars as a Destination in a Capability-Driven Framework
}

\author{
S. J. Hoffman, Ph.D ${ }^{1}$, B. G. Drake ${ }^{2}$, J. D. Baker ${ }^{3}$, S. A. Voels, Ph.D ${ }^{1}$ \\ ${ }^{1}$ Science Applications International Corporation, 2450 NASA Parkway, Houston, \\ Texas 77058; PH 281.483.9264 or 281.244.8171; email: stephen.j.hoffman@saic.com \\ or stephen.a.voels@saic.com \\ ${ }^{2}$ NASA Johnson Space Center, Code YA, 2101 NASA Parkway, Houston, Texas \\ 77058; PH 281.483.1806; email: bret.g.drake@nasa.gov \\ ${ }^{3}$ Jet Propulsion Laboratory, 4800 Oak Grove Drive, Pasadena, California 91109; PH \\ 818.354.5004; email: john.d.baker@jlp.nasa.gov
}

\section{ABSTRACT}

This paper describes NASA's current plans for the exploration of Mars by human crews within NASA's Capability-Driven Framework (CDF). The CDF describes an approach for progressively extending human explorers farther into the Solar System for longer periods of time as allowed by developments in technology and spacecraft systems. Within this framework, Mars defines the most challenging objective currently envisioned for human spaceflight.

The paper first describes the CDF and potential destinations being considered within this framework. For destinations relevant to the exploration of Mars, this includes both the Martian surface and the two moons of Mars. This is followed by a brief review of our evolving understanding of Mars to provide the context for the specific objectives set for human exploration crews. This includes results from robotic missions and goals set for future Martian exploration by NASA's communitybased forum, the Mars Exploration Program Analysis Group (MEPAG) and the MEPAG-sponsored Human Exploration of Mars - Science Analysis Group (HEMSAG).

The paper then reviews options available for human crews to reach Mars and return to Earth. This includes a discussion of the rationale used to select from among these options for envisioned Mars exploration missions.

The paper then concludes with a description of technological and operational challenges that still face NASA in order to be able to achieve the exploration goals for Mars within the CDF.

\section{INTRODUCTION}

Recently NASA's approach for future human exploration beyond low-Earth orbit has undergone extensive review and the current direction is less specific than that which NASA has been following for almost a decade (Anon., February 2009). Instead of a detailed plan aimed at future missions to the Moon and then to Mars (Anon., February 2009), a broader, less destination specific framework has emerged. This strategy, referred to as a Capability Driven Framework (Anon., January 12, 2011), is based on the idea of an ever expanding human presence beyond low-Earth orbit measured in terms of mission duration and distance from the Earth. It is based 
on evolving capabilities that are utilized for more challenging missions after operational experience has been established from less demanding missions. In theory, the Capability Driven Framework enables multiple destinations and provides increased flexibility, greater cost effectiveness, and sustainability. But the utility of a Capability Driven Framework can only be measured and fully understood when put into context of actual missions. Thus, to help formulate the strategies, technologies, and systems needed to support the framework, example destinations are being examined including low-Earth orbit, geostationary missions, cis-lunar space (including lunar fly-by, lunar orbit, and lunar surface), near-Earth asteroids, as well as missions to the surface of Mars and the moons of Mars. Before describing Mars missions in detail, a brief review of all the missions associated with the CDF is instructive.

Geostationary Orbits (GEO). This mission class includes missions to GEO or other high-Earth orbit destinations generally for the purpose of deploying or repairing ailing spacecraft. Due to the high delta- $\mathrm{V}$ associated with these destinations, a splitmission approach is typically used in which the crew is sent to the destination after cargo assets to be used for this mission arrive at the location where they will be used. The advantage of prepositioning cargo not needed by the crew until they arrive at the destination typically derives from the use of a more mass efficient trajectory/ propulsion system combination but at the expense of longer transit times. The cargo assets can include habitats, mobility systems, robotic systems, and repair equipment.

Earth-Moon Libration. This mission class includes missions to the EarthMoon L1 or L2 points or high lunar orbit. As with the GEO mission, cargo for these missions is sent separately from the crew. L1 can serve as an assembly point (or operational location) for large science platforms, or as a staging node for other destinations such as to the lunar surface, NEAs, or perhaps even Mars. Thus, crew missions to L1 may serve as the initial crew transport leg at the beginning or end of a different mission class.

Lunar Surface. Missions to the lunar surface will encompass a range of mission durations, beginning with short stays to prove the performance of the systems, to longer duration test beds for more challenging missions such as the surface of Mars. As with both the GEO and L1 missions, a split mission approach is typically used separating the crew from cargo.

Near-Earth Asteroids. This mission class represents human missions to and from asteroids which are in close proximity to Earth, orbit perihelion typically less than 1.3 Astronomical Units (AU). Near-Earth asteroids are of interest in this context because they represent a class of missions which truly leave Earth's vicinity. Since these missions are conducted in heliocentric space and the orbits of NEAs have long synodic periods, it becomes very difficult to pre-deploy mission assets prior to the crew mission. Thus, these missions are typically constructed as all-up missions, whereby all of the required mission assets are transported with the crew (e.g., deep space habitat, destination exploration systems, and Earth entry vehicle).

Mars Orbit. This mission class includes missions to the moons of Mars (Phobos and Deimos) as well as Mars orbit. Mission opportunities to Mars occur approximately every 26 months. Because these missions do not include planetary surfaces, the crew is exposed to the deep space environment for the entire mission 
duration. Mission opportunities occur on a frequent basis and thus pre-deployment of mission exploration assets is usually employed.

Mars Surface. This mission class represents missions to the surface of Mars. Strategies for exploring the surface of Mars typically utilize pre-deployed cargo vehicles and flying lower energy conjunction class missions. Details of this type are consistent with the NASA Mars Design Reference Architecture 5.0 (Drake, 2009).

Key features of all of these CDF mission classes are summarized in Table 1.

Table 1. Key Characteristics of Capabilities-Driven Framework Missions

\begin{tabular}{|c|c|c|c|c|c|c|}
\hline & $\begin{array}{c}\text { Geostationary } \\
\text { Orbit }\end{array}$ & $\begin{array}{l}\text { Earth-Moon } \\
\text { Libration }\end{array}$ & $\begin{array}{l}\text { Lunar } \\
\text { Surface }\end{array}$ & $\begin{array}{l}\text { Near-Earth } \\
\text { Asteroids (2) }\end{array}$ & $\begin{array}{l}\text { Mars Orbit } \\
\text { (Phobos) }^{(2)}\end{array}$ & $\begin{array}{c}\text { Mars } \\
\text { Surface }\end{array}$ \\
\hline \multicolumn{7}{|l|}{ Typical Mission Design } \\
\hline In-Space Delta-v $(\mathrm{km} / \mathrm{s})$ & 5.9 & 4.8 & $\sim 5.6$ & $4.0-9.0+$ & $\sim 9.0-15.0$ & $5.5-7.3$ \\
\hline Descent/Ascent or Vicinity Delta- $v(\mathrm{~km} / \mathrm{s})$ & - & - & 4.2 & - & $1.7-3.5$ & 6.3 \\
\hline Total Mission Duration (days) $)^{(3)}$ & 10 & 16 & $16-180$ & 365 & 660 & 900 \\
\hline Outbound Time (days) (s) & 0.5 & 4 & 5 & 170 & 250 & 180 \\
\hline Time at Destination (days)(s) & 9 & 8 & $7-180$ & 25 & 60 & 540 \\
\hline Return Time (days) (a) & 0.5 & 4 & 4 & 170 & 350 & 180 \\
\hline Crew Mission Mode & Zero-g & Zero-g & Zero-g & Zero-g (?) & Artificial-g & Zero-g \\
\hline Cargo Mode & Split & Split & Split & All-up & Split & Split \\
\hline Typical Mission Opportunities & Daily & $\begin{array}{l}\text { Weekly- } \\
\text { Monthly }\end{array}$ & $\begin{array}{l}\text { Weekly- } \\
\text { Monthly }\end{array}$ & $\begin{array}{c}10-50+ \\
\text { Years }\end{array}$ & $\begin{array}{l}\text { Every } 26 \\
\text { Months }\end{array}$ & $\begin{array}{l}\text { Every } 26 \\
\text { Months }\end{array}$ \\
\hline Quick Abort to Earth Availability & Anytime & Anytime & $\begin{array}{l}\text { Nearly } \\
\text { Anytime }\end{array}$ & None & None & None \\
\hline \multicolumn{7}{|l|}{ Typical Systems Required } \\
\hline Orion Multi Purpose Crew Vehicle & r & r & $\checkmark$ & $\checkmark$ & $\checkmark$ & $\checkmark$ \\
\hline Heavy Lift Launch (Space Launch System) & $\checkmark$ & 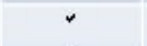 & 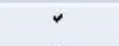 & $\checkmark$ & 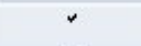 & 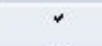 \\
\hline In-Space Propulsion & $\checkmark$ & r & $\checkmark$ & $\checkmark$ & 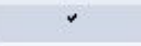 & 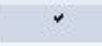 \\
\hline Destination Exploration Systems & $\checkmark$ & v & $\checkmark$ & $\checkmark$ & v & $\checkmark$ \\
\hline Deep-Space Habitat & & r & & * & r & 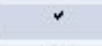 \\
\hline Planetary Lander & & & $\checkmark$ & . & & $\checkmark$ \\
\hline \multicolumn{7}{|l|}{ Key Technologies } \\
\hline Cryogenic Propulsion & r & r & r & r & 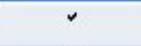 & r \\
\hline Radiation Protection & & & 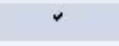 & $\checkmark$ & r & $\checkmark$ \\
\hline Advanced Propulsion (SEP, NEP, NTR) & & & & r & $\checkmark$ & r \\
\hline Near-Zero Boil off Cryogenic Fluid Storage & & & & $\checkmark$ & v & $\checkmark$ \\
\hline High-speed Earth Entry & & & & 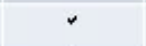 & v & $\checkmark$ \\
\hline Life Support System Enhancements & & & & r & r & - \\
\hline Zero-g Countermeasures & & & & $\checkmark$ & r & s \\
\hline In-Situ Resource Utilization & & & $\checkmark$ & & & $\checkmark$ \\
\hline Entry, Descent and Landing & & & 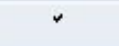 & & & $\checkmark$ \\
\hline Nuclear Surface Power & & & & & & 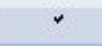 \\
\hline \multicolumn{7}{|l|}{ Typical Launch Parameters } \\
\hline \# SLS launches to send crew to destination & 1 & 1 & 1 & $2-3+(4)$ & $3-7^{(4)}$ & $3^{(4)}$ \\
\hline \# SLS launches for destination cargo & 1 & 1 & 1 & - & $2-3^{(*)}$ & $4^{(*)}$ \\
\hline Approximate total mass in LEO $(t)$ & 200 & 200 & 200 & $200-300^{(*)}$ & $500-900^{(4)}$ & $800^{(4)}$ \\
\hline $\begin{array}{l}\text { Notes: } \\
\text { (1) Dependent on the specific target chosen } \\
\text { (2) Typical for opposition class (short stay) } \mathrm{m} \\
\text { (3) Typical, but can be shorter or longer depe } \\
\text { (4) Assumes advanced propulsion }\end{array}$ & $\begin{array}{l}\text { issions and will var } \\
\text { ending on the targ }\end{array}$ & $\begin{array}{l}\text { y significantlyb } \\
\text { t chosen, time }\end{array}$ & $\begin{array}{l}y \text { mission op } \\
\text { pent at the }\end{array}$ & $\begin{array}{l}\text { ortunity } \\
\text { estination, and }\end{array}$ & total delta-v & \\
\hline
\end{tabular}




\section{EARTH-MARS MISSION PLANNING}

Round-trip missions between Earth and Mars are, in effect, a double rendezvous problem. The outbound trajectory from Earth must be established while considering the position of Mars at the end of this trajectory arc. Upon arrival at Mars the Earth is in a relatively unfavorable alignment (phase angle) for an energy efficient return. This unfavorable alignment results in two distinct classes of round-trip Mars missions: Opposition class missions, which are also commonly referred to as short-stay missions, and Conjunction class missions, referred to as long-stay missions. Practical considerations, such as total propulsive requirements, mission duration, surface objectives, and human health and safety must be considered in the mission design process when choosing between these mission classes. The synodic cycle, or mission repetition rate for identical Earth-Mars phasing and therefore launch opportunities for similar mission classes, is on the order of every 26 months. The mission characteristics such as mission duration, trip times, and propulsive requirements vary due to the eccentricity of Mars' orbit.

Opposition Class: Short-Stay Missions. Short-stay missions get this name because of the short stay-times (typically 30 - 60 days) at Mars. This is also referred to as an opposition-class mission. These short stay times are a consequence of orbit mechanics. Because of the relative positions of Earth and Mars at the time of Mars arrival, the optimal time to return to Earth has already passed and every additional day spent at Mars increases the difficulty (i.e., the amount of propellant needed) of the return trajectory. Round-trip mission times range from 550-660 days. A typical trajectory profile for a shortstay missions is shown in Figure 1. This class of mission has high propulsive requirements for the reasons mentioned previously. Shortstay missions always have one short transit leg, either outbound or inbound, and one long transit leg, the latter requiring close passage by the sun (0.7 AU or less). After arrival at Mars, rather than

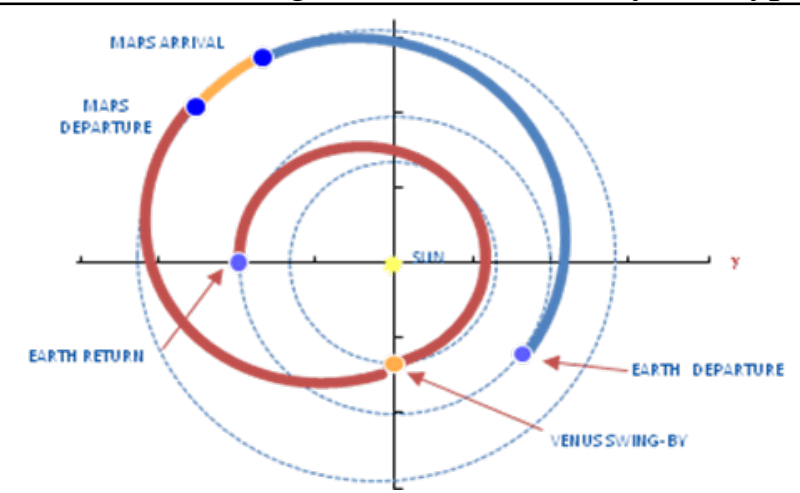

Figure 1. A typical opposition class trajectory between Earth and Mars. waiting for a near-optimum return alignment, the spacecraft initiates the return after a brief stay and the return leg cuts well inside the orbit of the Earth to make up for the non-optimal alignment of the planets that existed at Mars departure. Distinguishing characteristics of the short-stay mission are: 1) short-stay at Mars, 2) medium total mission duration, 3) perihelion passage inside the orbit of Venus on either the outbound or inbound legs, and 4) large total energy (propulsion) requirements.

Conjunction Class: Long Stay Missions. The second Mars mission class is typified by long-duration stay-times (as much as 550 days) at Mars and long total round-trip times (approximately 900 - 1000 days). This mission type (see Figure 2) is often referred to as conjunction-class, although the exploration community has 


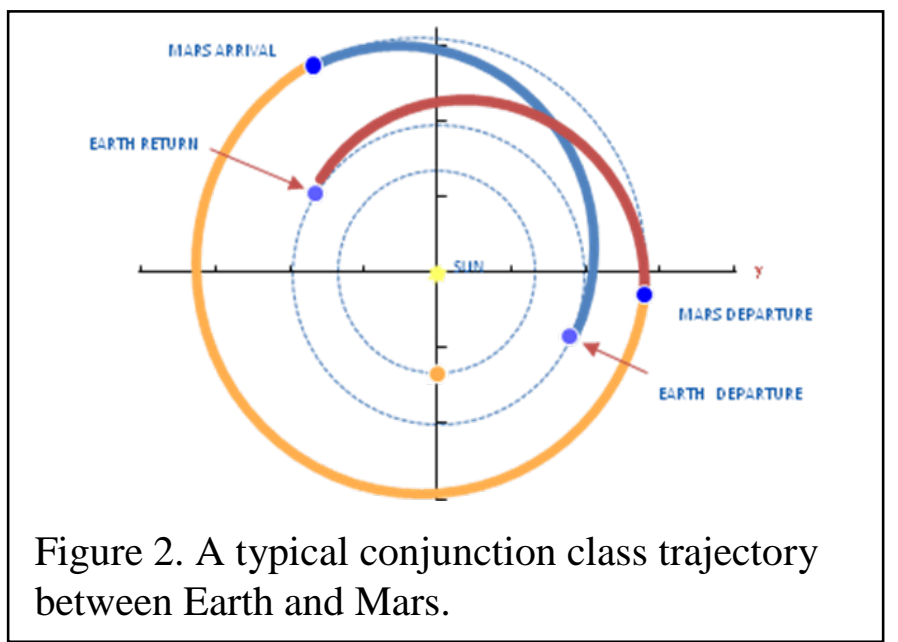

of the long-stay mission include: 1) lon Mars, 3) relatively little energy change between opportunities, 4) bounding of both transfer arcs by the orbits of Earth and Mars (closest perihelion passage of $1 \mathrm{AU}$ ), and 5) relatively short transits to and from Mars (less than 200 days).

\section{MARS EXPLORATION MISSIONS}

Human exploration of Mars has been a long-standing goal of human spaceflight and the subject of a great number of proposed approaches for accomplishing this goal. Two classes of Mars exploration missions are being carried in the CDF: missions in which the crew remains in orbit focused on understanding the two Martian moons or teleoperating robotic devices on the surface and missions in which the crew lands on the surface for an extended period of direct exploration.

Robotic Missions. There have been a steady set of exploration missions to Mars since 1997 to further understand its history, climate, and whether or not life may have existed. While seemingly Earth-like based on its current rotation period of 24.5 hours and many similar geological features to that on Earth, the planet is characterized as cold and dry. However, recent discoveries by both NASA and ESA probes have demonstrated that there were once long standing bodies of water on Mars early in its history. This was shown conclusively by the Mars Exploration Rover missions (Spirit and Opportunity) which landed in 2003. There is also recent evidence of water flow events on the surface as well. Furthermore, the ESA Mars Express spacecraft has detected the presence of methane in the Martian atmosphere and has shown that it is seasonal. The source of the methane is currently unknown.

While much is known about the Martian surface, there is much more to learn before sending humans. The MEPAG has identified precursor measurements needed in advance of human exploration. Currently the Mars Science Laboratory rover named 'Curiosity' is the next mission to address these goals and will determine if life ever existed on the surface, to further characterize the geological history, and characterize the climate. In addition to the wealth of geological, meteorology and chemical data that will be returned, two dedicated payloads were included to prepare for human exploration, the first will measure the high energy radiation environment 
and the second will better characterize the upper atmospheric density during the entry phase of the mission. This will enable improved performance predictions and eventually lead to landing larger vehicles on the surface.

Mars Orbital Missions. Because there are two distinct trajectory types for a round trip mission to Mars, there are also two distinct approaches for conducting exploration missions while in mars orbit. In both cases the crew of four spends the entire mission in the deep space environment and, likely, in a zero-gravity condition. Thus, in one respect there is an incentive to construct these missions to reduce crew exposure by flying the trajectories as fast as possible (i.e., short stay class trajectories) within the constraints of the propulsion technologies and number of heavy lift launches. On the other hand, using the longer duration trajectories (i.e., the long stay class) will definitely reduce the number of launches (for a fixed payload mass) but at the expense of increasing the crew's exposure to the deep space and zero-gravity environment. To reduce zero-gravity affects, the suggestion has been made to fly in an artificial-gravity (i.e., spinning) mode if possible (although there is as yet no conclusive evidence that artificial-gravity completely mitigates zero-gravity effects or does not introduce other deleterious effects on the crew). These factors are still being discussed and evaluated and, as a consequence, two different approaches to Mars orbital exploration are included in the CDF.

The first approach for Mars orbital exploration uses the short stay class of trajectories in order to minimize the total duration of the mission and thus the crew's exposure to the deep space and zero-gravity environment. A graphical view of the activities in this approach is shown in Figure 3. The short stay trajectory class limits the time spent in orbit around Mars, in this case to only 60 days. The large interplanetary spacecraft used to transport the crew to and from Mars is placed into a one-sol elliptical parking orbit where it rendezvous with cargo placed in this parking orbit on a previous transfer opportunity. Half of the crew uses a Space Exploration

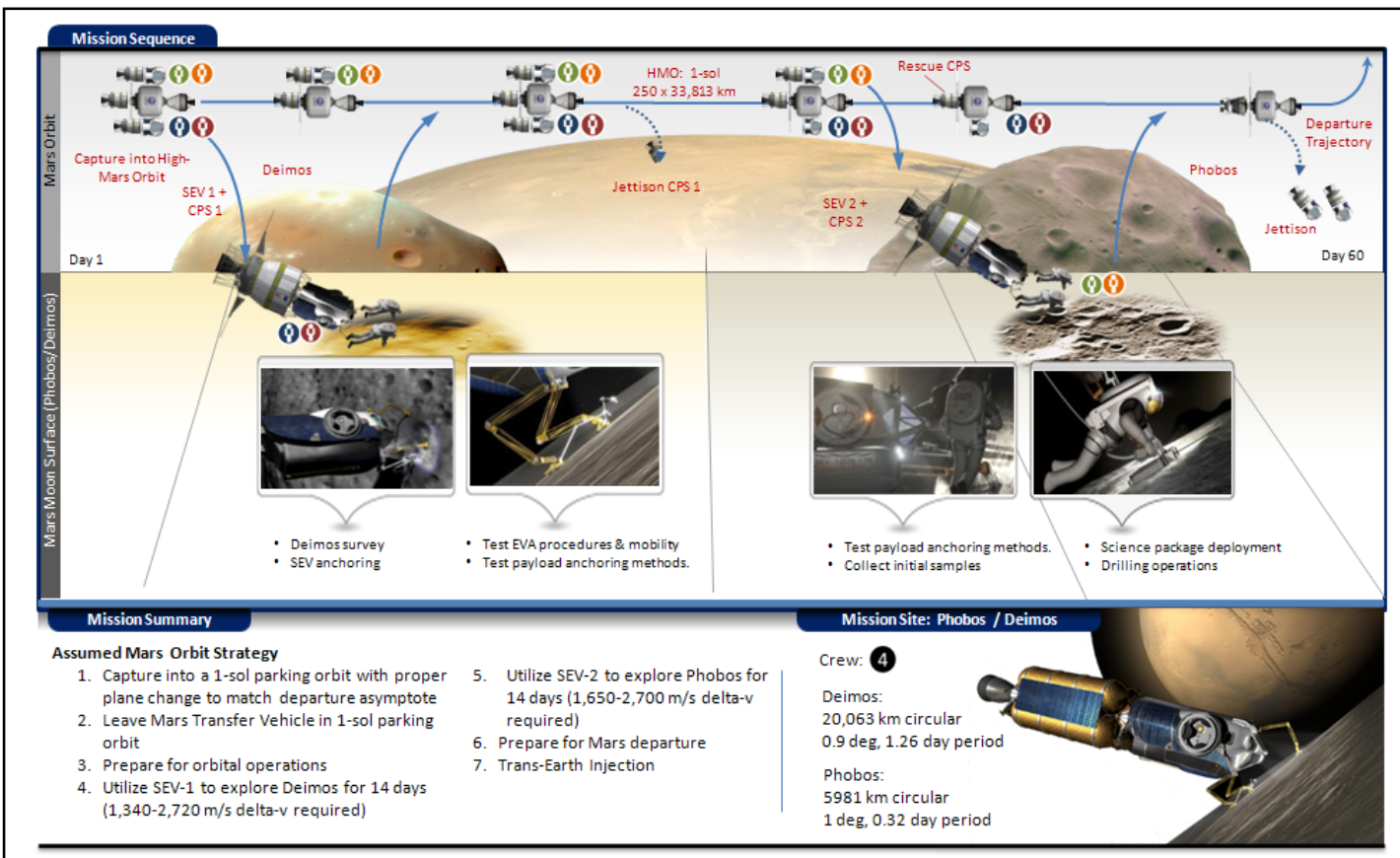

Figure 3. Short stay class of Mars orbital exploration. 
Vehicle (SEV) and one of three cryogenic propulsion stages (CPS) to transfer from this parking orbit to the vicinity of Deimos and spends the next two weeks exploring this moon. After returning from this Deimos mission, the other two crew members use a second SEV and CPS to transfer from the parking orbit to the vicinity of Phobos and spends the next two weeks exploring the other Martian moon. A third CPS is available to rescue either crew should they become stranded at either Phobos or Deimos. Crew time not used to explore Phobos or Deimos will be available to teleoperate robotic on the surface of Mars when a communication path is available. At the end of the 60-day stay all SEV and CPS assets are jettisoned and the large interplanetary spacecraft departs from its parking orbit for the return trip to Earth.

The second approach for Mars orbital exploration uses the long stay class of trajectories in order to maximize the amount of time available for exploring the Martian moons and any teleoperation of robots on the Martian surface. A graphical view of the activities in this approach is shown in Figure 4. Again the large interplanetary spacecraft used to transport the crew of four to and from Mars is placed into a one-sol elliptical parking orbit where it will rendezvous with cargo placed in this parking orbit on a previous transfer opportunity. Because of the duration spent in orbit around Mars in this scenario using a strategy similar to the short stay mission and deploying the crew to Phobos and Deimos for just a few weeks of exploration is not an effective use of the crew. The efficiency if the long stay transfer trajectory means that there is now enough propellant available for the large interplanetary vehicle, with its much larger habitat, to transfer into a parking orbit in the vicinity of Deimos, allowing the crew to spend an extended period of time (as necessary) exploring this moon using one of two SEVs (the second SEV is available for rescuing the first). There will still be sufficient propellant for the large interplanetary vehicle to then transfer to a parking orbit in the vicinity of Phobos, where the crew again spends an extended period of time (as necessary) exploring this moon. This approach

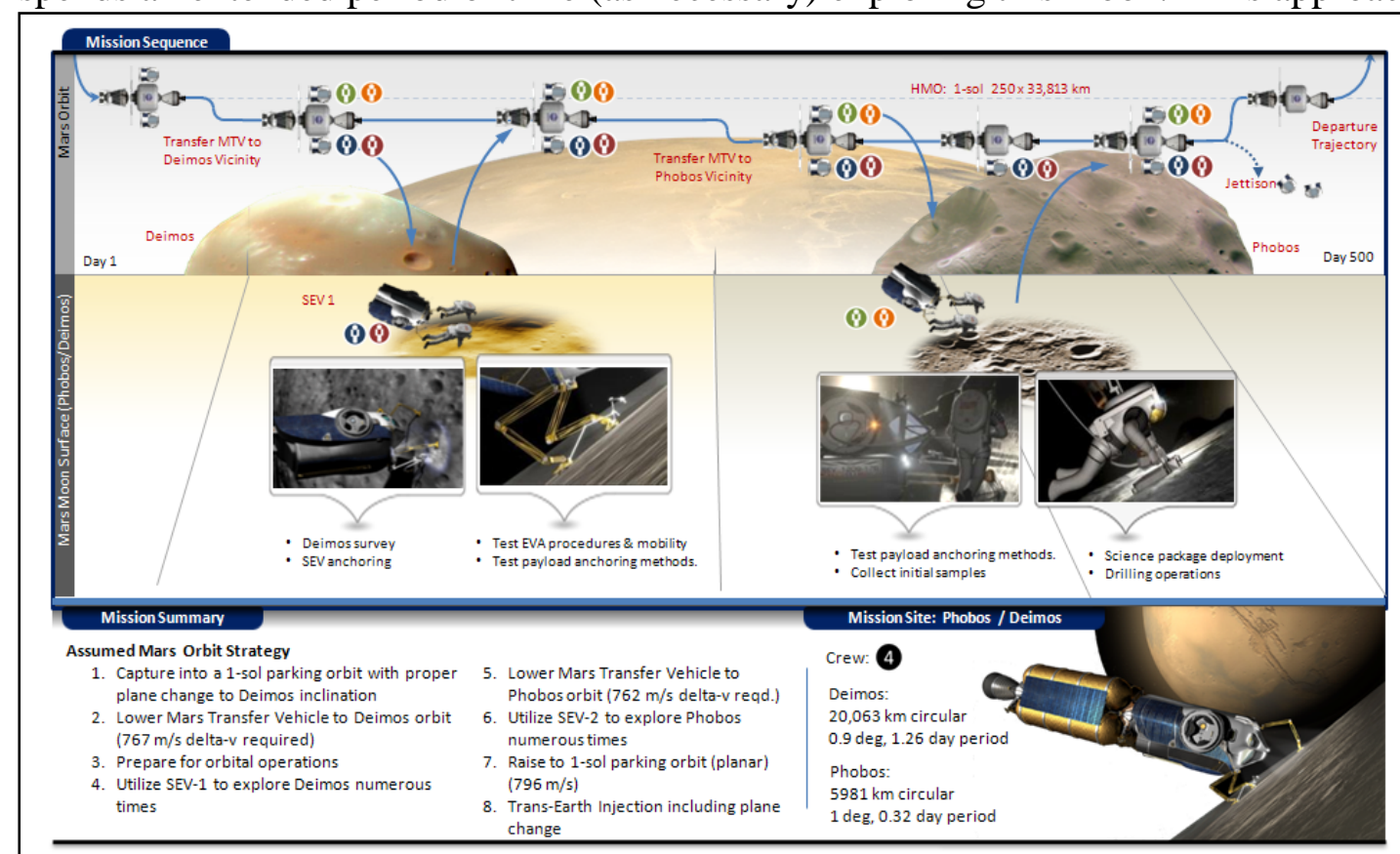

Figure 4. Long stay class of Mars orbital exploration. 
to exploring both of the Martian moons means that a separate CPS is not needed. The long stay class of orbits allows the crew to spend approximately 500 days exploring these two moons or teleoperating robots on the surface of Mars when a communication path is available.

Mars Surface Mission. A significant effort has been invested by NASA over the past quarter century examining different alternatives for human exploration missions of the surface of Mars. The culmination of these analyses have been captured in the NASA Design Reference Architecture 5.0 (Drake, 2009) which forms the basis for this CDF mission. A graphical view of the activities in this approach is shown in Figure 5. For this mission a crew of six is sent to Mars on a long stay class trajectory, the transfer portion of which is designed to last six months. On arrival the crew places their large interplanetary vehicle into a one-sol elliptical parking orbit to rendezvous with one of two cargo vehicles sent to Mars on the prior orbit transfer opportunity. The second cargo vehicle will have already landed at the intended surface exploration location where automated systems will have set up a power plant and a propellant manufacturing plant. Once the crew lands at this site they spend

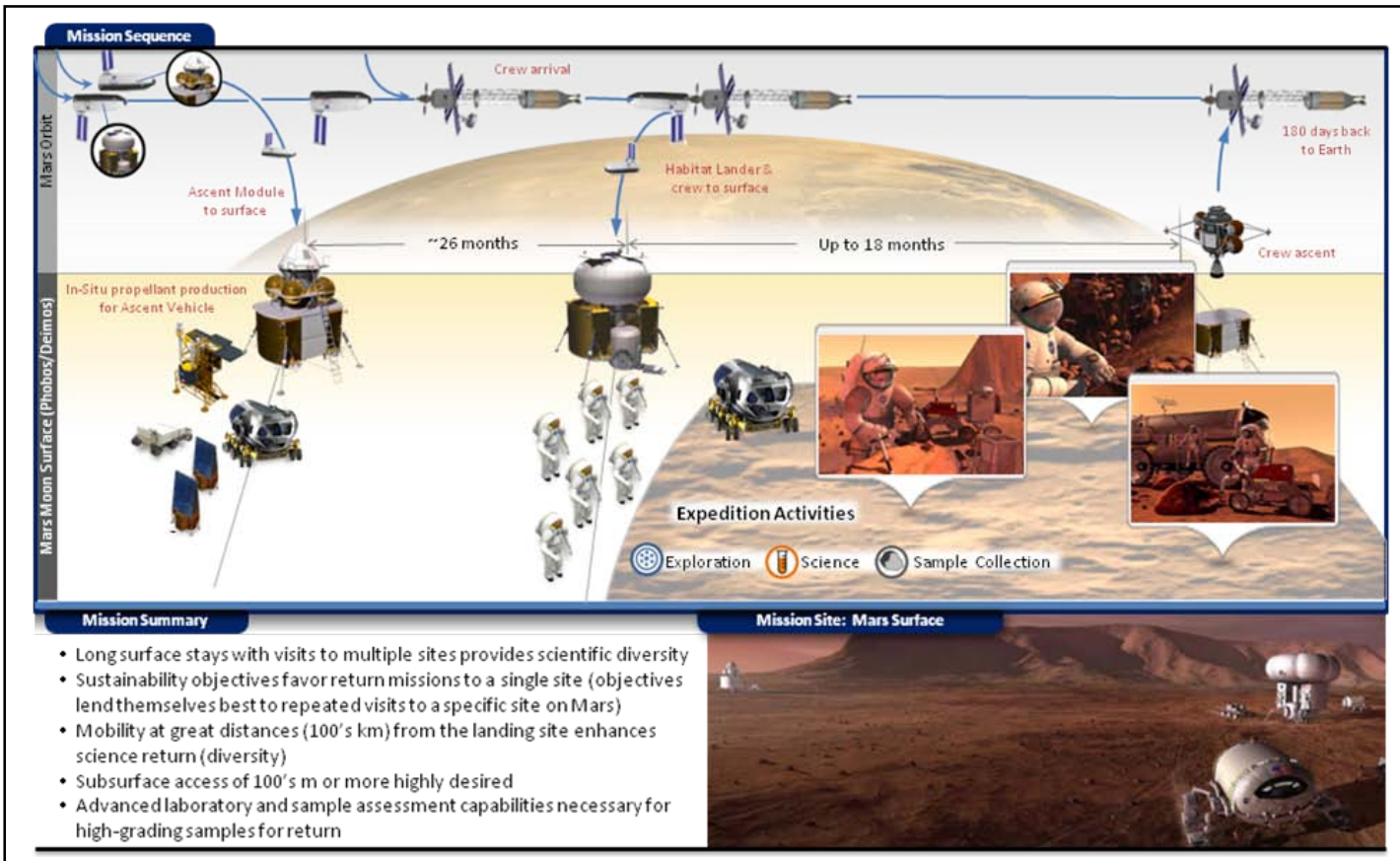

Figure 5. Mars surface exploration.

approximately 500 days exploring the vicinity in a series of long (at least 100 kilometer) traverses radiating from this fixed central base - an approach dubbed the “commuter" strategy in DRA 5.0 (Drake, 2009). At the completion of this surface mission the crew ascends from their surface base using propellants manufactured there and returns to the waiting interplanetary vehicle. At the appropriate time the crew departs from Mars for a six-month transfer back to Earth.

Details regarding a Mars surface reference mission continue to evolve as the understanding of Mars evolves. However a reasonably complete picture of this surface mission can be described at this time. The science community has stated three 
broad goals that are used to guide both robotic and human exploration of the surface (MEPAG, 2010):

1. Determine if life ever arose on Mars;

2. Understand the processes and history of climate on Mars;

3. Determine the evolution of the surface and interior of Mars.

No specific sites have been selected for human surface missions. The science community has stated that "progress would be optimized by visiting multiple sites and by maximizing the stay time at those sites" (Drake, 2009). Candidate sites will be selected based on the best possible data available at the time of the selection, the operational difficulties associated with the site, as well as the collective merit of the science and exploration questions that can be addressed at the site. Data available for site selection will include remotely gathered data sets plus data from any landed mission(s) in the vicinity plus interpretive analyses based on these data. From an operational perspective, a candidate site will likely have a relatively broad, relatively flat, centrally-located area where the surface systems can land in relative safety. However this places these systems and the crew at large distances from those features that are of interest to the crew and the science teams. These large distances coupled with the extended period of time the crew will stay in the surface implies that the crew will be given the capability to rove over distances of 10's to 100's of kilometers away from the landing site and spend days to weeks completing a round-trip traverse. Understanding the vertical structure of the site will also be of interest, indicating that a drilling capability will be included for each mission and site. The ability to move this drill form location to location will also be desirable.

\section{CONCLUSION: TECHNICAL AND OPERATIONAL CHALLENGES}

NASA has recently embarked on a different path for the future exploration of the inner solar system by human crews. At the current stage in its development this different path, the Capability Driven Framework, is being framed in terms of a series of Design Reference Missions each of which is focused on a specific destination but in the aggregate describe a building block approach to expanding human presence in the solar system. This paper has described the most challenging of the DRMs that are currently part of the CDF - the human exploration of Mars. Table 1 indicates where many of these challenges will occur. This table indicates that a substantial number of key technological advances will be necessary to make the implementation of this mission a reasonable endeavor. Figure 6 illustrates how improvements in these key technologies will favorably impact the overall scale of the Mars surface mission; similar improvements can be expected for Mars orbital missions. But even with these technological improvements the mission parameters shown in Table 1 indicates that operational challenges remain. Mars missions are likely to have some of the longest durations of any mission within the CDF. The large distances involved will increase round-trip communication times to as much as 40 minutes, changing the role of a traditional Earth-based mission control to more of a mission support function and increasing the level of autonomy under which the crew must operate. And even with 
one of the largest launch vehicles ever developed, Mars missions will require a substantial number of successful launches for each mission, placing greater emphasis on developing highly reliable systems, carefully processing those systems for flight, and a highly choreographed launch campaign. But Table 1 also indicates how the CDF, with the current suite of DRMs, can address and resolve most of these challenges with a properly sequenced program of increasingly demanding scenarios.

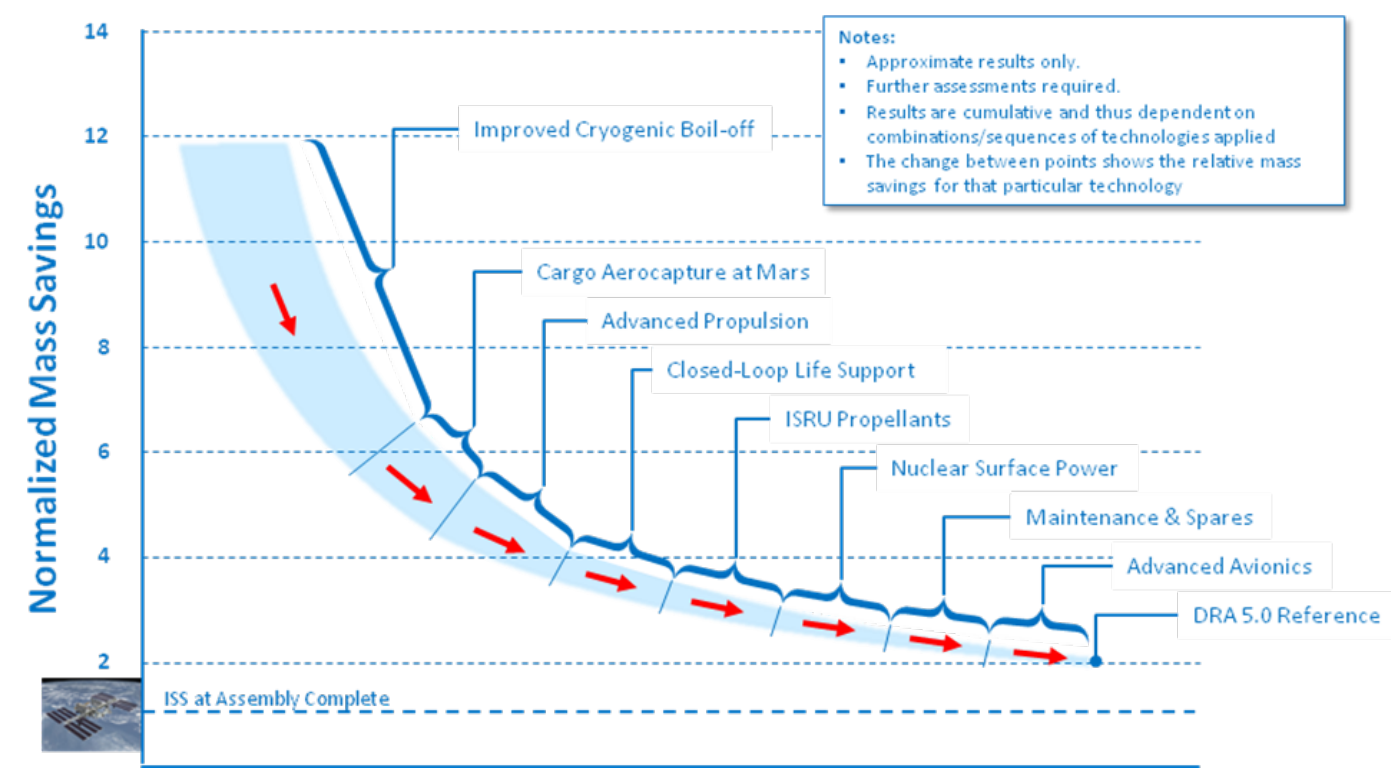

Figure 6. Technology improvements that will enable Mars missions.

\section{REFERENCES}

Anon. (February 2009). “The Vision for Space Exploration”, NP-2004-01-334HQ, National Aeronautics and Space Administration.

Anon. (January 12, 2011). "Human Space Exploration Framework Summary”, National Aeronautics and Space Administration, http://www.nasa.gov/exploration/new_space_enterprise/home/heft_summary.html

Drake, Bret G. (July 2009). Human Exploration of Mars, Design Reference Architecture 5.0, NASA/SP-2009-566, National Aeronautics and Space Administration, Washington, DC.

MEPAG (September 2010). "Mars Science Goals, Objectives, Investigations, and Priorities," Mars Exploration Program Analysis Group, National Aeronautics and Space Administration, Washington, DC. 